\title{
P-type Doping of Epitaxial 3C-SiC Layers on Silicon (001)
}

\author{
G. Wagner ${ }^{1, a}$, M. Schmidbauer ${ }^{1, b}$, K. Irmscher ${ }^{1, c}$, P. Tanner ${ }^{2, d}$ \\ and R. Fornari ${ }^{1, e}$ \\ ${ }^{1}$ Leibniz-Institute for Crystal Growth, Max-Born-Str. 2, D-12489 Berlin, Germany \\ ${ }^{2}$ Griffith University, Nathan, Queensland, Australia, 4111 \\ awagner@ikz-berlin.de, bschmidbauer@ikz-berlin.de, cirmscher@ikz-berlin.de, \\ efornari@ikz-berlin.de, ${ }^{d}$ p.tanner@griffith.edu.au
}

Keywords: 3C-SiC, hot-wall-CVD, heteroepitaxy, p-type doping, TMA

\begin{abstract}
Single-crystalline 3C-SiC epitaxial layers were grown on on-axis $\mathrm{Si}(001)$ substrates by low-pressure hot-wall chemical vapour deposition. Aluminium from a trimethylaluminium (TMA) source was used for p-type doping. The atomic Al and carrier concentrations in the layers were determined as a function of the partial pressure of the TMA source gas. Secondary ion mass spectroscopy (SIMS), Hall-effect measurements at room temperature and four-point electrical resistivity method were applied to measure the atomic and electrically active Al concentrations. The crystalline perfection of the layers was characterized by high-resolution x-ray diffraction (HRXRD). At TMA-partial pressures ranging from $5 \times 10^{-7}$ mbar up to $1.5 \times 10^{-4}$ mbar corresponding aluminium concentrations from $2 \times 10^{15} \mathrm{~cm}^{-3}$ up to $1.3 \times 10^{19} \mathrm{~cm}^{-3}$ were measured in the epitaxial layers. On increasing the Al concentration from $1 \times 10^{17} \mathrm{~cm}^{-3}$ to $1 \times 10^{19} \mathrm{~cm}^{-3}$ the layer electrical resistivity decreases from $17 \Omega \mathrm{cm}$ to $0.8 \Omega \mathrm{cm}$, while no influence on the crystalline quality of the layers was observed. The average full width at half maximum (FWHM) of the rocking curve for a $5 \mu \mathrm{m}$ thick 3C-SiC layer is about 500 arcsec. With increasing layer thickness (up to $16 \mu \mathrm{m}$ ) the FWHM of the rocking curve decreases to about 300 arcsec.
\end{abstract}

\section{Introduction}

Aluminium is a major p-type dopant in silicon carbide. For hexagonal polytypes $(4 \mathrm{H}-$ and $6 \mathrm{H}-\mathrm{SiC})$ high-quality p-type layers (aluminium dopant) have been successfully grown and the carrier concentrations could be accurately tuned for the development of SiC devices [1-3]. On the other hand, there are only few investigations about incorporation of p-type dopants in the cubic polytype. Only a limited number of articles about successful p-type doping of 3C-SiC have been published [4-7]. The epitaxial growth of cubic $\mathrm{SiC}$ with high crystalline quality is strongly dependent on the quality of the seed layer on the carbonized Si (001) surface. A variety of experimental methods to create cubic $\mathrm{SiC}$ seed layers on $\mathrm{Si}$ substrates and to deposit 3C-SiC epitaxial layers with low defect density have been published [8-10]. To exploit such layers for fabricating $\mathrm{SiC}$ devices for both electronic and micro/nano-mechanical applications, n-type and p-type doping of the layers is required. In this article the solubility of aluminium in $3 \mathrm{C}-\mathrm{SiC}$ epitaxial layers grown by hot wall CVD is investigated.

\section{Experiment}

The heteroepitaxial growth of 3C-SiC layers on on-axis 2-inch $\mathrm{Si}(001)$ substrates (thickness 275 $\mu \mathrm{m})$ was carried out in a low-pressure hot-wall chemical vapour deposition (CVD) reactor. A detailed description of the CVD-system is given elsewhere [5]. The reaction gases, $\mathrm{SiH}_{4}$ and $\mathrm{C}_{3} \mathrm{H}_{8}$ diluted in hydrogen, are carried by palladium-purified $\mathrm{H}_{2}$. Before loading them into the reactor the Si (001) substrates were cleaned by using the standard RCA procedure followed by immersion in a HF solution in order to remove the surface oxide. The carbonisation of the Si surface before layer deposition was identified as a most critical step, independent of the deposition temperature. The 
carbonisation of the $\mathrm{Si}$ surface started with introduction of $13 \mathrm{sccm} \mathrm{C}_{3} \mathrm{H}_{8}$ at a temperature lower than $800{ }^{\circ} \mathrm{C}$ immediately followed by a heating ramp up to the growth temperature of $1350{ }^{\circ} \mathrm{C}$. Additionally, a small amount of $\mathrm{SiH}_{4}$ (partial pressure $\mathrm{SiH}_{4}$ : $5 \times 10^{-3} \mathrm{mbar}, \mathrm{C} / \mathrm{Si}$ ratio: 40 ) was added to the input gas mixture to prevent evaporation of Si from the substrate and to avoid the formation of voids at the interface between the substrate and layer when the substrate reached a temperature of about $975^{\circ} \mathrm{C}$. This carbonisation process produced a nucleation layer consisting of $3 \mathrm{C}-\mathrm{SiC}$ crystallites of about $20 \mathrm{~nm}$ in size. The subsequent 3C-SiC layer deposition took place at a temperature of $1350^{\circ} \mathrm{C}$ under a reactor pressure of $150 \mathrm{mbar}$. The silane flow was adjusted to produce a deposition rate of $2.5 \mu \mathrm{m} / \mathrm{h}$. The $\mathrm{C} / \mathrm{Si}$ ratio in the gas phase was fixed at 2, and the hydrogen carrier gas flow was $30 \mathrm{slmin}^{-1}$ in the different deposition experiments. In order to obtain p-type resistivity trimethylaluminium (TMA) was added into the gas atmosphere with partial pressures from $5 \times 10^{-7}$ mbar up to $1.5 \times 10^{-4}$ mbar.

The atomic Al concentrations were analyzed quantitatively using secondary ion mass spectroscopy (SIMS) while the electrically active carrier concentration was determined by Hall-effect measurements at room temperature. The electrical resistivity of the layers was measured by a fourpoint method. High-resolution x-ray diffraction (HRXRD) investigations were carried out in order to obtain valuable information about the crystalline quality and residual stress in the p-type $3 \mathrm{C}$-SiC layers.

\section{Results}

The formation of a homogeneous few-nanometre thick and flat 3C-SiC-buffer layer on the Sisubstrate is an important prerequisite to obtaining an epitaxial cubic SiC-layer with high structural perfection. The $\mathrm{SiH}_{4}$ partial pressure and the $\mathrm{C} / \mathrm{Si}$ ratio during the carbonisation of the Si-surface and the heat up procedure to the deposition temperature have to be optimized according to the hydrogen carrier gas flow rate and the final deposition temperature. Management of the Si-partial pressure in the gas atmosphere inhibited the appearing of voids or inclusions at the interface between the substrate and layer. Fig. 1 shows the photograph of the interface between the Si-substrate and a $5 \mu \mathrm{m}$ thick $3 \mathrm{C}-\mathrm{SiC}$ epitaxial layer in Nomarski differential interference contrast (NDIC). Because the layer is transparent in NDIC-contrast it is possible to observe the interface and voids if they exist. In fig. 1 only boundaries between (001) oriented 3C-SiC domains are visible. The formation of voids was inhibited.

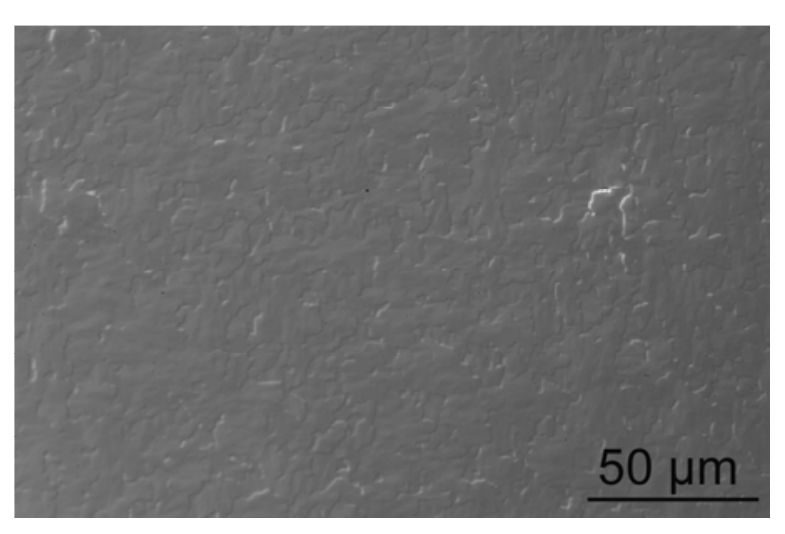

Fig.1: Nomarski differential interference contrast (NDIC) micrograph of a $5 \mu \mathrm{m}$ thick 3C-SiC layer on a $\mathrm{Si}(001)$-substrate.

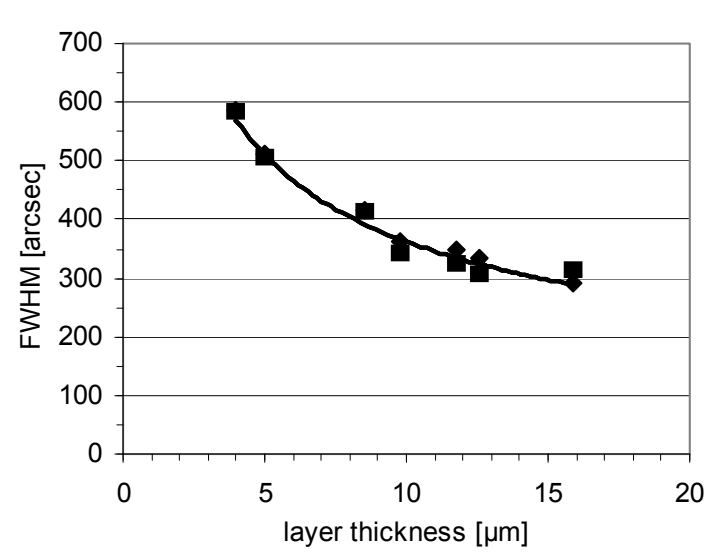

Fig.2: FWHM of the HRXRD rocking curve vs. the 3C-SiC layer thickness, $(\bullet(004), \boldsymbol{\square}(002)-X R D-r e f l e c t i o n)$

Undoped 3C-SiC layers always showed n-type conduction. Nitrogen, which contaminates the growth cell during loading under laboratory atmosphere, is responsible for the unintentional n-type 
doping. Dependent on the baking out procedure after loading the growth cell immediately before the carbonisation-deposition process, the n-type doping level can shift. The samples analysed in this work have a background nitrogen concentration of about $1.5 \times 10^{15} \mathrm{~cm}^{-3}$.

To obtain p-type conduction, TMA with partial pressures from $4 \times 10^{-7}$ mbar up to $1 \times 10^{-4} \mathrm{mbar}$ was added to the growth atmosphere. In this range of TMA concentrations no influence on the growth rate and the layer surface properties was detected. The thickness of the $3 \mathrm{C}$-SiC layers was proportional to the growth time. The typical growth rate was $2.5 \mu \mathrm{m} /$ hour, which was obtained setting a silane partial pressure of $3 \times 10^{-2} \mathrm{mbar}$ in the growth atmosphere. The layer surface was smooth and mirror-like with a roughness of about $8 \mathrm{~nm}$.

The crystallinity of the layers was characterized by HRXRD. The full width at half maximum (FWHM) of the 3C-SiC (002) and (004) reflection for a layer thickness of $5 \mu \mathrm{m}$ is about 500 arcsec. For increasing thickness the FWHM decreases, which indicates an increasing crystalline perfection. Owing to the large difference in the lattice parameter between $\mathrm{Si}$ and 3C-SiC (20\%) a high density of dislocations is present in the first $500 \mathrm{~nm}$ of the grown layer. With increasing layer thickness the dislocation density is strongly suppressed. The dependence of the FWHM on the layer thickness is shown in fig. 2. With increasing thickness from $5 \mu \mathrm{m}$ to $16 \mu \mathrm{m}$ the FWHM decreases from 500 arcsec to about 300 arcsec. An influence of the increasing Al content on the FWHM of the rocking curve of the layers was not observed.

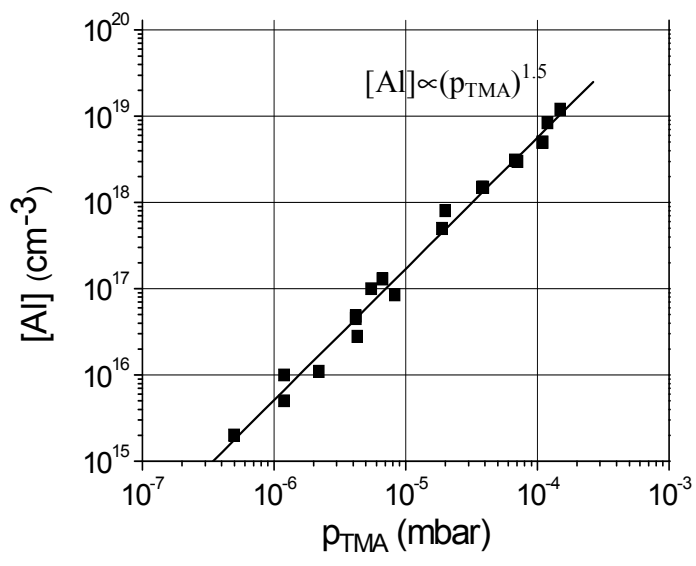

Fig. 3: Dependence of Al concentration in 3C-SiC layers on the TMA-partial pressure in the growth atmosphere.

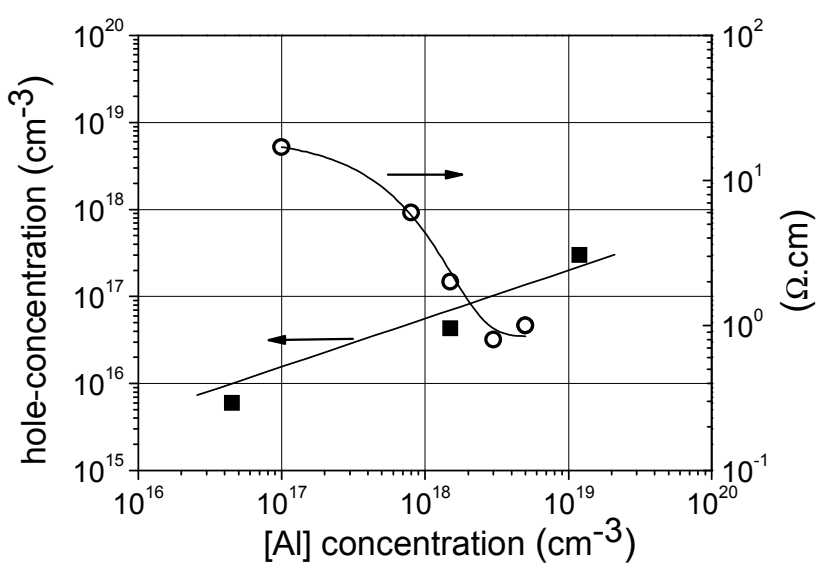

Fig.4: Carrier concentration and electrical resistivity vs. Al concentration in $3 \mathrm{C}-\mathrm{SiC}$ layers

Fig. 3 shows the results of SIMS measurements of the Al concentration as a function of the partial pressure of the TMA source. The Al concentration increased over proportional with increasing TMA partial pressure $\left(\mathrm{p}_{\mathrm{TMA}}\right),[\mathrm{Al}] \propto\left(\mathrm{p}_{\mathrm{TMA}}\right)^{1.5}$, for the applied C/Si ratio of 2. At TMA-partial pressures from $5 \times 10^{-7}$ mbar up to $1.5 \times 10^{-4}$ mbar the concentration of atomic aluminium increased from $2 \times 10^{15} \mathrm{~cm}^{-3}$ up to $1.3 \times 10^{19} \mathrm{~cm}^{-3}$. Fig. 4 shows the results of the Hall effect and the four point resistivity measurements at room temperature and the dependence on the Al concentration. With increasing atomic Al concentration from $4.5 \times 10^{16} \mathrm{~cm}^{-3}$ to $1.3 \times 10^{19} \mathrm{~cm}^{-3}$ the hole-concentration increases from $6 \times 10^{15} \mathrm{~cm}^{-3}$ to $3 \times 10^{17} \mathrm{~cm}^{-3}$, while the layer resistivity decreases from $17 \Omega \mathrm{cm}$ to $0.8 \Omega \mathrm{cm}$, respectively. The ratio of the hole concentration to the total Al concentration is about 0.02. This difference is mainly caused by the high ionization energy of the $\mathrm{Al}$ acceptor $(0.24 \mathrm{eV})$. Furthermore the $\mathrm{Al}$ atoms may be trapped at dislocations and domain boundaries, making them electrically inactive. 


\section{Summary}

Single-crystalline p-type $3 \mathrm{C}$-SiC epitaxial layers have been grown on on-axis $\mathrm{Si}(001)$ substrates by low-pressure hot-wall chemical vapour deposition. Trimethylaluminium was used as the Al-source. The dependence of the atomic and electrically active Al concentration on TMA partial pressure at a growth temperature of $1350{ }^{\circ} \mathrm{C}$ and a process pressure of 150 mbar were investigated. The $\mathrm{Al}$ concentration increased over proportional with increasing TMA partial pressure $\left(\mathrm{p}_{\mathrm{TMA}}\right),[\mathrm{Al}] \propto\left(\mathrm{p}_{\mathrm{TMA}}\right)^{1.5}$, for the applied C/Si ratio of 2 .

An atomic aluminium concentration from $2 \times 10^{15} \mathrm{~cm}^{-3}$ up to $1.3 \times 10^{19} \mathrm{~cm}^{-3}$ at a TMA-partial pressure from $5 \times 10^{-7} \mathrm{mbar}$ up to $1.5 \times 10^{-4} \mathrm{mbar}$ in to the source gas atmosphere has been measured by SIMS. At atomic Al-concentrations from $2 \times 10^{15} \mathrm{~cm}^{-3}$ up to $1.3 \times 10^{19} \mathrm{~cm}^{-3}$ the hole concentrations increase from $6 \times 10^{15} \mathrm{~cm}^{-3}$ to $3 \times 10^{17} \mathrm{~cm}^{-3}$ and the layer resistivity decreases from $17 \Omega \mathrm{cm}$ to $0.8 \Omega \mathrm{cm}$, respectively. The increasing Al-content has no influence on the crystalline quality of the layers. The average FWHM of the rocking curve for a $5 \mu \mathrm{m}$ thick $3 \mathrm{C}$-SiC layer is about 500 arcsec caused by the high density of misfit dislocations near the interface between the substrate and layer. With increasing layer thickness up to $16 \mu \mathrm{m}$ the FWHM of the XRD-curve decreases to about 300 arcsec.

\section{References}

[1] G. Wagner, W. Leitenberger, K. Irmscher, F. Schmid, M. Laube, G. Pensl: Materials Science Forum Vols. 389-393 (2002), p. 207

[2] U. Forsberg, O. Danielsson, A. Henry, M. K. Linnarsson, E. Janzen: J. Crystal Growth Vol. 250 (2003), p. 471

[3] G. Wagner, D. Schulz, D. Siche: Progress of Crystal Growth and Characterization of Materials Vol. 47 (2005), p. 139

[4] H. J. Kim, R. F. Davis: J. Electrochem. Soc. Vol. 133 (1986), p. 2350

[5] S. Nishino, H. Suhara, H. Ono, H. Matsunami: J. App. Phys. Vol. 61 (1987), p. 4889

[6] K. Takahashi, S. Nishino, J. Saraie: J. Crystal Growth Vol. 115 (1991), p. 617

[7] M. A. Capano, A. R. Smith, B. C. Kim, E. P. Kvam, S. Tsoi, A. K. Ramdas, J. A. Cooper: Materials Science Forum, Vol. 527-529 (2006), p. 431

[8] T. Chassagne, G. Ferro, D. Chaussende, F. Cauwetm, Y. Monteil, J. Bouix: Thin Solid Films Vol. 402 (2002), p. 83

[9] E. Bustarret, D. Vobornik, A. Roulot, T. Chassagne, G. Ferro, Y. Monteil, E. MartinezGuerrero, H. Mariette, B. Daudin, L. S. Dang: phys. stat. sol. (a) Vol. 195 (2003), p. 18

[10] J. Yun, T. Takahashi, T. Mitani, Y. Ispida, H. Okumura: J. Crystal Growth Vol. 291 (2006), p. 148 